\title{
Connecting VLBI and Gaia Celestial Reference Frames
}

\author{
Zinovy Malkin 1, 2,3*
}

${ }^{1}$ Department of Radio Astronomy Research, The Pulkovo Astronomical Observatory, St. Petersburg, Russia, ${ }^{2}$ Institute of Earth Sciences, St. Petersburg State University, St. Petersburg, Russia, ${ }^{3}$ Astronomy and Cosmic Geodesy Department, Kazan Federal University, Kazan, Russia

\section{OPEN ACCESS}

Edited by:

Ludwig Combrinck,

Hartebeesthoek Radio Astronomy Observatory, South Africa

Reviewed by:

Alberto Vecchiato, INAF - Astrophysical Observatory of Torino, Italy

Robert Heinkelmann, Helmholtz-Zentrum Potsdam Deutsches GeoForschungsZentrum GFZ, Germany

*Correspondence: Zinovy Malkin malkin@gao.spb.ru

Specialty section:

This article was submitted to Fundamental Astronomy,

a section of the journal Frontiers in Astronomy and Space

Sciences

Received: 07 April 2016

Accepted: 26 August 2016

Published: 12 September 2016

Citation:

Malkin Z (2016) Connecting VLBI and Gaia Celestial Reference Frames. Front. Astron. Space Sci. 3:28. doi: 10.3389/fspas.2016.00028
The current state of the link problem between radio and optical celestial reference frames is considered. The main objectives of the investigations in this direction during the next few years are the preparation of a comparison and the mutual orientation and rotation between the optical Gaia Celestial Reference Frame (GCRF) and the 3rd generation radio International Celestial Reference Frame (ICRF3), obtained from VLBI observations. Both systems, ideally, should be a realization of the ICRS (International Celestial Reference System) at micro-arcsecond level accuracy. Therefore, the link accuracy between the ICRF and GCRF should be obtained with similar error level, which is not a trivial task due to relatively large systematic and random errors in source positions at different frequency bands. In this paper, a brief overview of recent work on the GCRF-ICRF link is presented. Additional possibilities to improve the GCRF-ICRF link accuracy are discussed. The suggestion is made to use astrometric radio sources with optical magnitude to $20^{m}$ rather than to $18^{m}$ as currently planned for the GCRF-ICRF link. In addition, the use of radio stars is also a prospective method to obtain independent and accurate orientation between the Gaia frame and the ICRF.

Keywords: astrometry, reference systems, International Celestial Reference Frame (ICRF), Gaia Celestial Refernce Frame (GCRF), link between radio and optical frames

\section{INTRODUCTION}

The ESA's Gaia space astrometry mission (Perryman et al., 2001; Lindegren et al., 2008) commenced successfully in December 2013 and its main scientific program in July 2014. One of the most important results of the Gaia mission will be a new highly-accurate optical celestial reference frame; Gaia Celestial Reference Frame (GCRF). Although the final GCRF version is expected to be available in the early 2020s, intermediate releases are planned, the first of them (DR1) is expected to be released in 2016. A new release of the VLBI-based celestial reference frame of similar accuracy, the 3rd realization of the International Celestial Reference Frame (ICRF3) is planned for 2018 (Jacobs et al., 2014).

Both radio (ICRF) and optical (GCRF) frames must be realizations of the same concept of the International Celestial Reference System (ICRS), Arias et al. (1995) with an expected accuracy at the level of a few tens of $\mu$ as. The link between the ICRF and GCRF should be realized at a similar level of accuracy, which is not a trivial task. This problem is similar to that of the link between the Hipparcos Celestial Reference Frame (HCRF) and the ICRF (Kovalevsky et al., 1997). Generally speaking, both GCRF and ICRF object positions are time-dependent. Therefore, analogously to HCRF, both the orientation and rotation of the GCRF with respect to ICRF are to be defined. In this paper, the general term "orientation" is used to avoid a non-principal discussion related to spin. Interested readers can find more theoretical and practical details in Lindegren and Kovalevsky (1995) and Kovalevsky et al. (1997). 
On the one hand, the link task is more straightforward for the GCRF than for the HCRF, as most of the ICRF objects will be directly observed by Gaia. On the other hand, the task is much more complicated due to the requirement that a much higher level of accuracy for the GCRF-ICRF link is needed so as to not compromise the high level of precision of the two frames.

The basic method to tie the Gaia catalog to the ICRF, and hence to the ICRS, is using Gaia observations of compact extragalactic ICRF objects that have accurate radio astrometric positions. With the help of these common objects, the orientation angles between the ICRF and GCRF will be determined. Finally, the GCRF catalog will be aligned to the ICRS by applying these orientation angles. The accuracy of this link depends on many factors, such as random and systematic errors of both radio and optical catalogs.

The objective of this paper is to briefly overview recent work on the ICRF-GCRF link and to discuss new possibilities to improve the link accuracy. It should be noted that the link between the GCRF and ICRF is not a task currently planned for completion before the end of this decade (Jacobs et al., 2014). Based on the Hipparcos experience, it can be envisioned that the work on improving such a link will be continued for a prolonged period after completion of the Gaia mission. The improvements will be primarily based on a new VLBI-based celestial reference frame (CRF) realization of which the accuracy can improve over time. New after-mission Gaia data reductions are also possible. Therefore, research and development of the methods for the linking of radio and optical reference frames will remain one of the primary tasks of fundamental astrometry throughout the next decade.

The paper is structured as follows. In Section 2, basic equations used to link two CRF realizations are described. Section 3 contains a brief overview of recent investigations regarding aspects concerning the ICRF-GCRF link. The following three sections are devoted to a discussion of new possibilities and possible improvements in both theoretical analysis and the final ICRF-GCRF link accuracy, such as the choice of the ICRF realization used for modeling and simulation (Section 4), using more link sources (Section 5), using radio stars (Section 6), and proper accounting for the galactic aberration in proper motions (Section 7).

\section{BASIC EQUATIONS}

Each catalog of the positions of celestial objects (CRF realization), be it ICRF or GCRF, represents its own coordinate frame linked to the ICRS at some degree of accuracy. Mutual orientation between these frames is defined by the three orientation angles $A_{1}, A_{2}$, and $A_{3}$ around the three ICRS Cartesian axes. Since the catalogs under consideration are close to each other at sub-arcsecond level, the orientation of a vector $(X, Y, Z)$, can be written in the following simple form:

$$
\left(\begin{array}{c}
X_{1} \\
Y_{1} \\
Z_{1}
\end{array}\right)=\left(\begin{array}{ccc}
1 & A_{3} & -A_{2} \\
-A_{3} & 1 & A_{1} \\
A_{2} & -A_{1} & 1
\end{array}\right)\left(\begin{array}{l}
X_{2} \\
Y_{2} \\
Z_{2}
\end{array}\right)
$$

Taking into account that on the celestial sphere

$$
\left(\begin{array}{l}
X \\
Y \\
Z
\end{array}\right)=\left(\begin{array}{c}
\cos \alpha \cos \delta \\
\sin \alpha \cos \delta \\
\sin \delta
\end{array}\right)
$$

and turning to the differences between the object positions in two catalogs $\Delta \alpha=\alpha_{1}-\alpha_{2}$ and $\Delta \delta=\delta_{1}-\delta_{2}$, the final expression can be derived:

$$
\begin{aligned}
\Delta \alpha & =A_{1} \cos \alpha \tan \delta+A_{2} \sin \alpha \tan \delta-A_{3}, \\
\Delta \delta & =-A_{1} \sin \alpha+A_{2} \cos \alpha .
\end{aligned}
$$

The system of Equation (3) for all or selected common objects in two catalogs is solved by the least squares method (LSM) to determine the orientation angles $A_{1}, A_{2}$, and $A_{3}$ between two CRF realizations and their errors (uncertainties).

Generally speaking, the differences between the two catalogs include not only the rotational part but also other, mostly coordinate-dependent terms that describe the systematic errors in the compared catalogs, including distortion of the celestial frames realized by the catalogs. Determination of the systematic errors of the celestial object positions in catalogs is a traditional and well developed astrometric task, see, e.g., Sokolova and Malkin (2007) and papers cited therein. Since these systematic errors might influence the orientation angles, they should be estimated during the GCRF-ICRF alignment procedure.

\section{OVERVIEW OF RECENT ACTIVITY}

The GCRF astrometric catalog will join both galactic stars and extragalactic objects in a single highly-accurate system. As a next stage, this catalog will be aligned to the ICRS using common GCRF and ICRF objects. Two tasks should be solved to provide such an alignment, analogously to what was done for the Hipparcos catalog (Lindegren and Kovalevsky, 1995; Kovalevsky et al., 1997):

1. Determination of mutual orientation between the two frames at an initial epoch, most naturally at the Gaia mean observation epoch, which is expected to be $\sim 2017.0$ or somewhat later if the mission will be prolonged after its 5-year initially planned duration.

2. Determination of the mutual rotation between two systems.

For the Hipparcos catalog, the achieved accuracy was 0.6 mas at the epoch 1991.25 for orientation and 0.25 mas/yr for rotation (Kovalevsky et al., 1997). In the case of the GCRF, the desired accuracy is about an order better, which is quite a challenge.

On the outset, the accuracy of the link between GCRF and ICRF will depend on the number of common objects and their astrometric quality, i.e., accuracy of their coordinates in two catalogs. If Gaia just observes all the objects it can detect with nearly uniform accuracy over the sky depending mainly on the object's optical brightness, the situation with the ICRF sources is more complicated. The ICRF2 catalog is very inhomogeneous in respect to the radio source position errors due to the large difference in number of observations from 3 to 
337,322, see Section 4 for more detail. The situation has improved substantially with realization of the project on re-observation of the VCS (VLBA Calibrator Survey) sources (Gordon et al., 2016), which allowed a significant improvement in the accuracy of about 2000 ICRF source positions (see Section 4), but it is still far from ideal.

Current activities in preparation toward aligning the GCRF with the ICRF are developing in several directions:

1. Selection of prospective link radio sources and their intensive observations.

2. Photometric optical observations of radio sources.

3. Optical astrometric observations of radio sources with ground-based telescopes.

4. Creation of data banks of optical images of ICRF objects.

Several years ago, Bourda et al. (2008) started work on selecting optically bright radio sources of good radio astrometric quality, i.e., having sufficient flux density and compact structure. Finally, 195 prospective link sources were selected. The various stages of this work were described by Bourda et al. (2008) (selection of optically bright radio sources), Bourda et al. (2010) (source detection on the long VLBI baselines), Bourda et al. (2011) (source imaging to estimate their radioastrometric quality), Le Bail et al. (2016) (improving radio positions of selected sources). The program is being continued.

Zacharias and Zacharias (2014) obtained accurate optical positions of $413 \mathrm{AGN}$, and investigated their errors and their impact on the accuracy of the link between radio and optical frames. Comparison of the optical positions with radio positions showed that the differences statistically exceed the known errors in the observations. The physical offset between the optical and radio emission centers was identified as a likely cause. This effect, called by the authors detrimental, astrophysical, random noise (DARN), was found to be at $\sim 10$ mas level. The authors came to the conclusion that the GCRF-ICRF orientation angles can hardly be determined with an error better than 0.5 mas, without a substantial increase in the number of the link objects. This estimate was based on ground-based results and can be somewhat improved with Gaia observations, but the DARN can prevail in this case too.

The new catalog URAT1 contains positions of over 228 million objects in the magnitude range of about $R=3-18.5$ with a typical error of 10-30 mas and proper motions of over 188 million objects with a typical error of 5-7 mas/yr (Zacharias et al., 2015). There are two shortcomings of this catalog limiting its usefulness for studies on the GCRF-ICRF link. Firstly, the catalog covers only just over a half of the sky, namely the region with $\delta \geq 15^{\circ}$. Secondly, most of the astrometric radio sources are absent in the URAT1 catalog as they are fainter than $18.5^{\mathrm{m}}$ (see Figure 4). However, this work is of great importance as it provides very valuable information on the shift between radio and optical positions for hundreds of radio sources.

A dedicated program of photometric observations of the ICRF sources have been conducted under coordination of the Paris Observatory Taris et al. (2013, 2015, 2016). These observations provide much new information about the optical brightness of the astrometric radio sources as well as about their optical variability. The results reported by this group showed that the peak-to-peak change in optical magnitude is typically at a level of several tenths of magnitude, and exceed $1^{m}$ for many objects reaching sometimes $3^{m}$, e.g., for the source B0716+714.

Andrei et al. $(2012,2014)$ have been working on the compilation of the Gaia QSO catalog. Data from different surveys and catalogs are assembled to complete QSO characteristics including positions, photometry, morphology, and imaging. This work, in particular, provides useful data for investigation of the radio minus optics position shift. The most complete bank of optical images of the ICRF objects was created by Andrei et al. (2015).

Souchay et al. (2015) presented the 3rd release of the Large Quasar Astrometric Catalog (LQAC-3). This catalog contains accurate positions, magnitudes in 9 bands UBVGRIZJK, radio flux in 5 bands from $750 \mathrm{MHz}$ to $30 \mathrm{GHz}$, morphology indexes in $B R I$ bands, and absolute magnitude in $B$ and $R$ band for 321,957 objects, primarily quasars, including 5\% of other AGN types.

All these works are directed, in particular, toward better link source selection and the improvement in the accuracy of their radio astrometric positions, which provides better accuracy of the GCRF-ICRF link. In the next sections, new possibilities to improve the quality of the link will be discussed.

\section{RADIO FRAME}

All the recent studies discussed in Section 3 are based on using the ICRF2 as a radio CRF. However, the ICRF2 catalog created in 2009 (Fey et al., 2015) is already outdated. It is expected that the first link between the GCRF and ICRF will be performed during 2018-2019 using the next VLBI-based ICRF realization, ICRF3 (Jacobs et al., 2014). Currently, the radio source position catalog gsf2015b ${ }^{1}$ derived at the NASA Goddard Space Flight Center (GSFC) VLBI Group appears to be the closest to the future ICRF3. It is computed using about the same data analysis strategy as was used for computation of ICRF2 (also at GSFC) but involves many more observations (VLBI delays). Table 1 contains a statistical comparison between the gsf $2015 \mathrm{~b}$ and ICRF2 catalogs.

Figure 1 illustrates the position uncertainty distribution in two catalogs. One can see that the overall level of the position uncertainty in the gsf $2015 \mathrm{~b}$ catalog is much smaller than that in the ICRF2 catalog.

It can be seen from Table 1 and Figure 1 that the GSFC catalog is more advanced when compared with the ICRF2. It should be noted that the gsf2015b catalog provides the original position errors obtained from the LSM solution, while ICRF2 position errors were inflated following the formula $\sigma_{\text {inflated }}^{2}=$ $\left(1.5 \sigma_{\text {computed }}\right)^{2}+(0.04 \text { mas })^{2}$ (Fey et al., 2015), see Figure 2 . The error floor of 0.04 mas is mostly effective for small original errors less than 0.1-0.2 mas; for larger original errors inflated errors may be taken as original errors multiplied by factor 1.5. Nevertheless, even taking this factor into account, gsf2015b source positions are

\footnotetext{
${ }^{1}$ http://gemini.gsfc.nasa.gov/solutions/astro/.
} 
TABLE 1 | Basic statistics of the ICRF2 and gsf2015b catalogs.

\begin{tabular}{|c|c|c|c|c|c|c|}
\hline \multirow[t]{2}{*}{ Catalog } & \multirow[t]{2}{*}{ Sources } & \multirow[t]{2}{*}{ Sessions } & \multirow[t]{2}{*}{ Delays } & \multirow[t]{2}{*}{ Period } & \multicolumn{2}{|c|}{ Median error, $\mu$ as } \\
\hline & & & & & $\alpha \cos \delta$ & $\delta$ \\
\hline ICRF2 & 3414 & 4540 & $6,495,553$ & 1979.08.03-2009.03.16 & 397 & 739 \\
\hline gsf2015b & 4089 & 5836 & $10,453,527$ & 1979.08.03-2015.11.09 & 123 & 210 \\
\hline
\end{tabular}

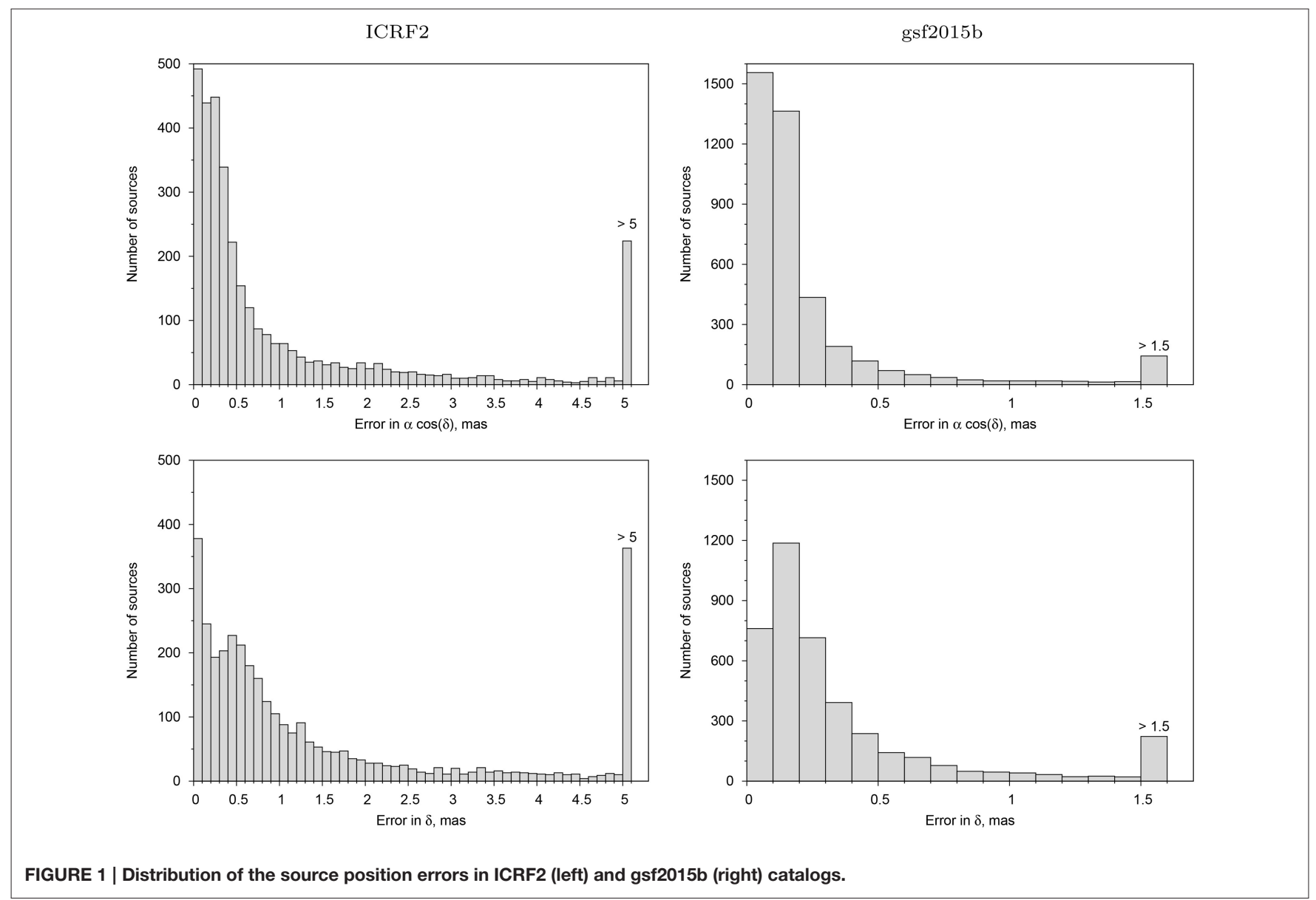

substantially more precise than those of the ICRF2. The primary reason for the significant improvement in source position errors is re-observation of about 2000 VCS (VLBA Calibrator Survey) sources (Gordon et al., 2016), which is $\sim 2 / 3$ of the total number of the ICRF2 sources.

Based on these considerations, the gsf $2015 \mathrm{~b}$ catalog was used in this study as a prototype of ICRF3.

One of the permanent tasks of the VLBI community is improving accuracy of the radio source positions. Due to limited VLBI resources, proper planning of the observations is important. Figure 3 illustrates the dependence of the source position uncertainty on the number of observations (delays and sessions) for the ICRF2 and gsf2015b catalogs. The error floor of 0.04 mas introduced in the final catalog (Fey et al., 2015) can clearly be seen in the plot for ICRF2. This analysis shows that the source position uncertainties depend primarily on the number of delays, and to lesser extent on the number of sessions. It also suggests that to reliably achieve sub-mas position error, about 100 observations (VLBI delays) should be obtained.

\section{INCREASING THE NUMBER OF LINK RADIO SOURCES}

A criterion for the initial source selection in Bourda et al. (2008), which was the base for the consequent works Bourda et al. (2010, 2011); Le Bail et al. (2016), was using ICRF2 sources with optical magnitude $\leq 18^{m}$. The latter limit was defined to use the objects with a small Gaia position error $<\sim 70 \mu$ as as was estimated during Gaia pre-launch analysis (Lindegren et al., 2008). To select the optically bright radio sources, the catalog of Véron-Cetty and Véron (2006) was used as the source of the photometric data.

It seems that the approach applied by Bourda et al. (2008) for the link source selection can be substantially improved in view 
of new data that became available during recent years. First, as was discussed in Section 4, the ICRF2 does not provide the best choice of astrometric radio sources with reliable highly-accurate

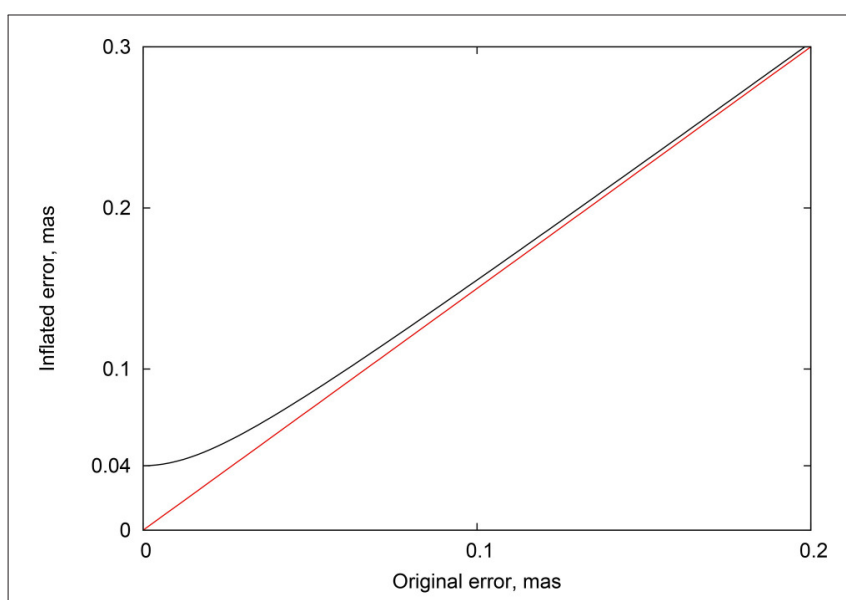

FIGURE 2 | Dependence of the inflated ICRF2 position errors on the original ones. Black line corresponds to the inflation formula $\sigma_{\text {inflated }}^{2}=\left(1.5 \sigma_{\text {computed }}\right)^{2}+(0.04 \text { mas })^{2}$ used in Fey et al. (2015). Red line corresponds to simple regression $\sigma_{\text {inflated }}=1.5 \sigma_{\text {computed }}$, i.e., to the ICRF2 formula with the error floor omitted. coordinates. Second, the catalog Véron-Cetty and Véron (2006) does not contain sufficiently complete photometric data as compared with the latest catalogs. So, an improved strategy for preliminary link source selection can be suggested. A new approach can include the use of the latest VLBI-based CRF solutions containing more radio sources with accurate positions, especially in the Southern Hemisphere, and the catalog OCARS (Optical Characteristics of Astrometric Radio Sources, Malkin, 2016b) that contains the most complete photometric data for astrometric radio sources and thus provides the maximum choice for selection of optically bright radio sources.

The second option that is worth investigating is using radio sources with a magnitude $18<G \leq 20^{m}$, where $G=20^{m}$ is the threshold for Gaia observations (Perryman et al., 2001; Lindegren et al., 2008). Although fainter sources will have much larger Gaia positional error, the number of these sources may compensate for such a loss of precision. An analysis based on the rotation covariance matrix analysis was performed by Mignard (2014). It was found that moving from $18^{m}$ to $20^{m}$ threshold leads to about a twofold increase of ICRF2 link sources from $\sim 500$ to $\sim 1000$, and reduction of the errors of the orientation angles from $\sim 7 \mu$ as to $\sim 6 \mu$ as.

Another approach based on Monte Carlo simulation was used in the current study. A list of the link sources for these computations was composed of all AGN with the optical

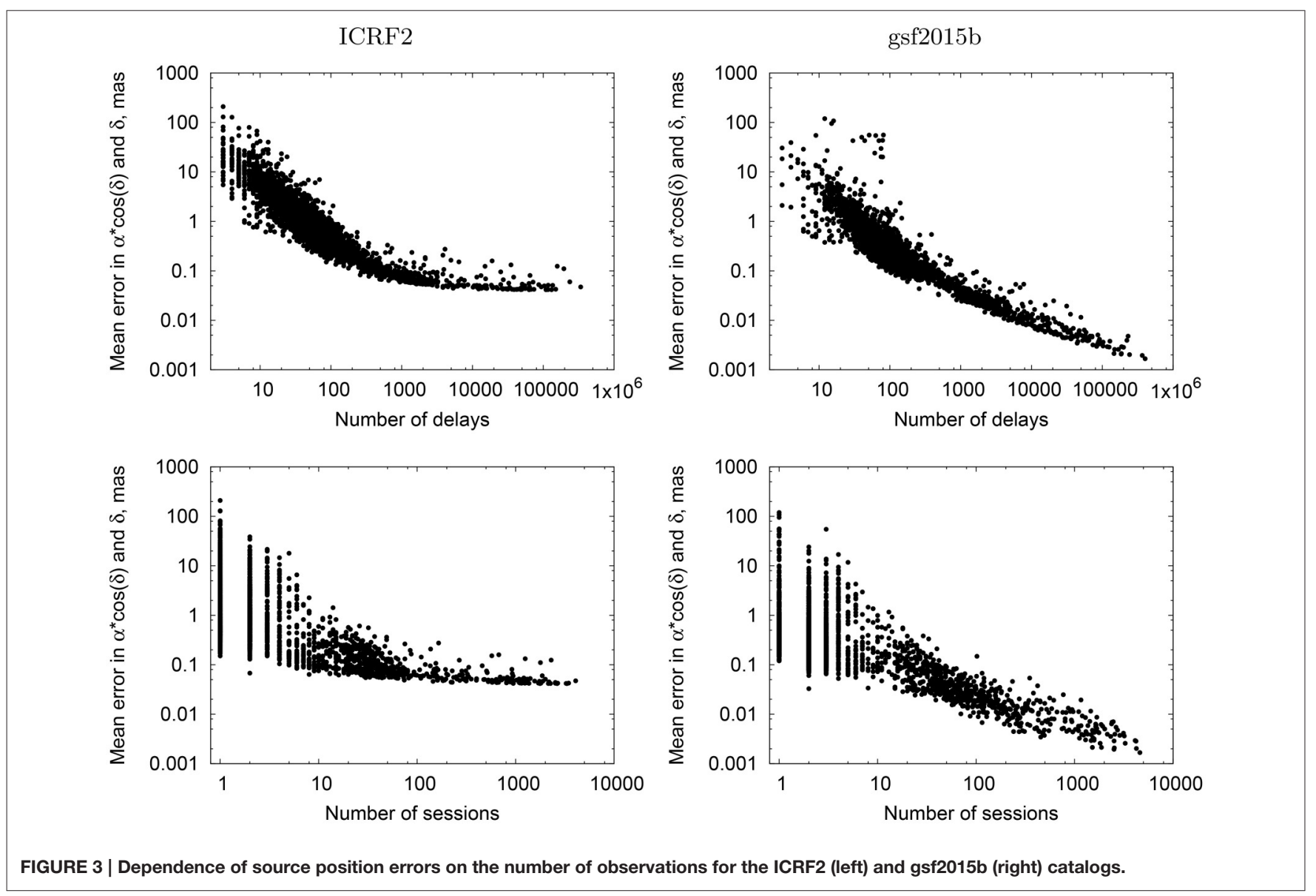


magnitude $\leq 20^{m}$ and galaxies with the optical magnitude $\geq 16^{m}$ selected from the gsf2015b catalog. It is supposed that faint galaxies can be expected to be unresolved objects for Gaia (de Souza et al., 2014). Optical magnitudes for these sources were taken from the OCARS catalog (Malkin, 2016b). Figure 4 illustrates the distribution of the optical magnitudes in the source set used in analysis.

The median uncertainty of the gsf2015b source positions for different sets of sources selected with different upper limits for the optical magnitude is depicted in Figure 5. One can see that moving to the optically fainter sources improves the overall position precision.

For the VLBI position errors, the uncertainty estimates given in the gsf $2015 \mathrm{~b}$ catalog were used. The Gaia position errors used during Monte Carlo simulation were estimated in the following way. Expected Gaia parallax standard error $\sigma_{\pi}$ depending on the optical brightness of the object $G$ is given by de Bruijne et al. (2014) (points in Figure 6). The authors also provide a rather complicated formula representing $\sigma_{\pi}$ depending on the optical

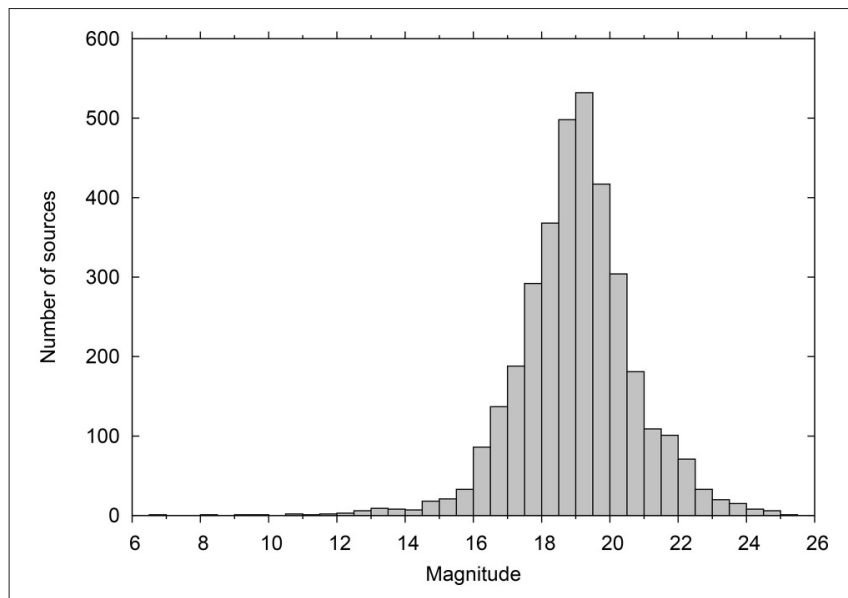

FIGURE 4 | Distribution of optical magnitudes in the gsf2015b catalog.

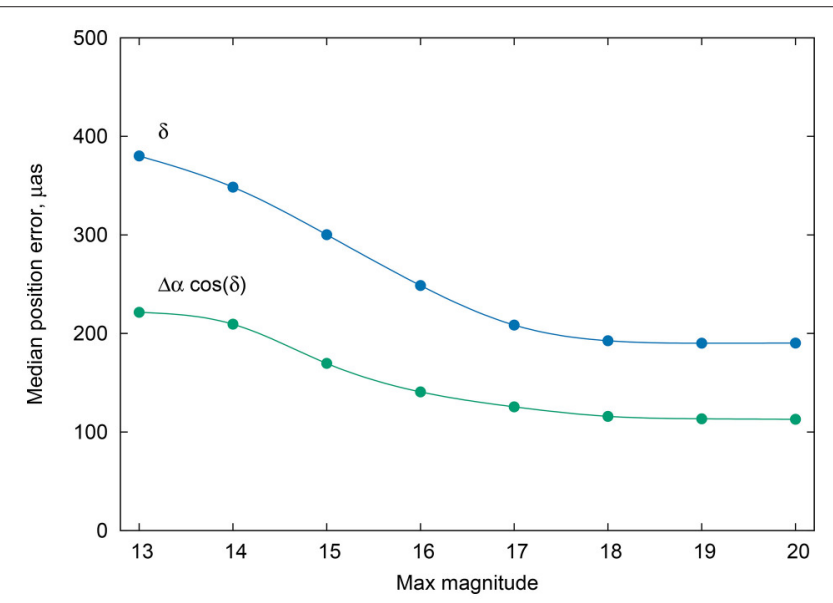

FIGURE 5 | Median errors of the gsf2015b positions depending on source selection based on the faint-end optical magnitude threshold. brightness. However, this formula appears to be unnecessarily complicated for the simulation studies, it depends not only on the $G$ magnitude but also on the $V-I$ color, which is not known for most of the astrometric radio sources. So, a simpler approximation of the $\sigma_{\pi}$ was derived (in $\mu$ as):

$\sigma_{\pi}= \begin{cases}6.7, & G<12.1, \\ 6.7+4.86(G-12.1), & 12.1 \leq G<13, \\ 10^{\left(1.044+0.1528(G-13)+0.01373(G-13)^{2}\right)}, & G \geq 13 .\end{cases}$

The Gaia position error is computed as $\sigma_{0}=0.743 \sigma_{\pi}$ (de Bruijne et al., 2014). This approximation function is depicted in Figure 6.

The result obtained with Monte Carlo simulation (10,000 iterations) is shown in Figure 7. This result is generally similar to that obtained by Mignard (2014) using a different method,
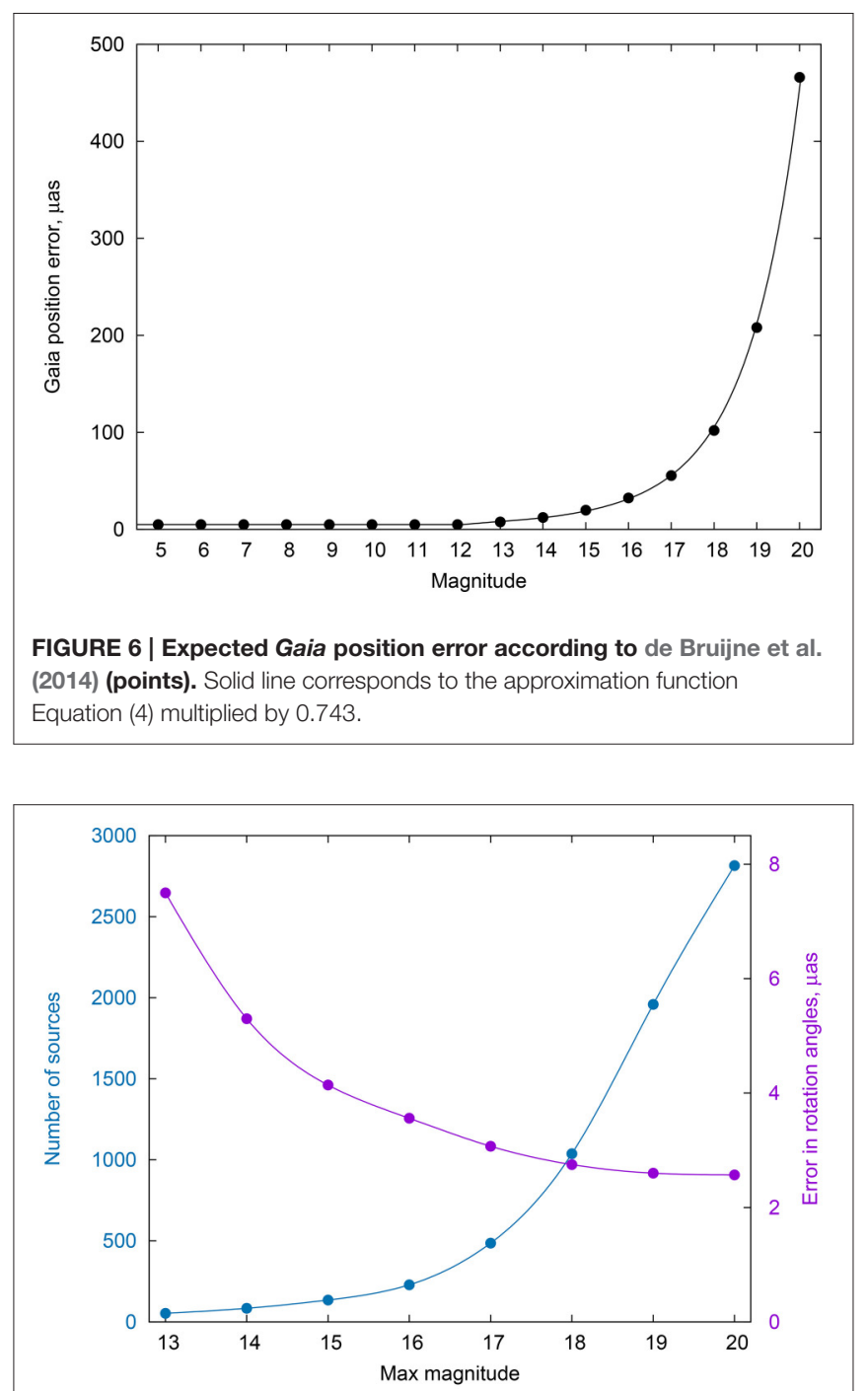

FIGURE 7 | Simulated error in orientation angles depending on source selection based on the faint-end optical magnitude threshold. 
however the errors in the orientation angles are about two times smaller in the current work, in particular, due to larger number of sources used.

Surely, both results are too optimistic because they were obtained without taking into account the radio-optics position shift, such as the DARN mentioned in Section 3. To estimate the impact of this effect, an additive error $\sigma_{\text {added }}$ was added in quadrature to the simulated ICRF-GCRF coordinate differences. The result of this test obtained by Monte Carlo simulation in the same way as the previous one is presented in Figure 8. The obtained error in the orientation angles agrees with the value of 0.5 mas predicted by Zacharias and Zacharias (2014) for $\sigma_{\text {added }}=$ 10 mas.

Finally, all the results presented in this section showed that it is advisable to use all compact radio sources up to $20^{m}$ for the ICRF-GCRF link. The distribution of 2815 gsf2015b sources with the optical magnitude $\leq 20^{m}$ over the sky is shown in Figure 9. Indeed, this is the first stage of selection of the best ICRF-GCRF link sources that should follow by estimation of their radioastrometric quality as discussed in Bourda et al. (2008). However, it allows one to have several times more prospective link sources at this stage than was considered earlier: 2815 sources vs. 535 sources selected by Bourda et al. (2008).

\section{USING RADIO STARS}

The problem of aligning the GCRF to ICRF is similar to the problem of aligning the HCRF to the ICRF. One of the methods applied for this purpose during the Hipparcos mission was using radio stars. It proved to be the most accurate method among others considered for the orientation of the Hipparcos catalog (Kovalevsky et al., 1997). The error in the orientation angles between the two frames obtained from VLBI observations of 12 radio stars was estimated to be about 0.5 mas.

Later, Boboltz et al. (2007) reported on the results of observations of 46 radio stars with flux density of 1-10 mJy obtained with the VLA (Very Large Array) plus the Pie Town

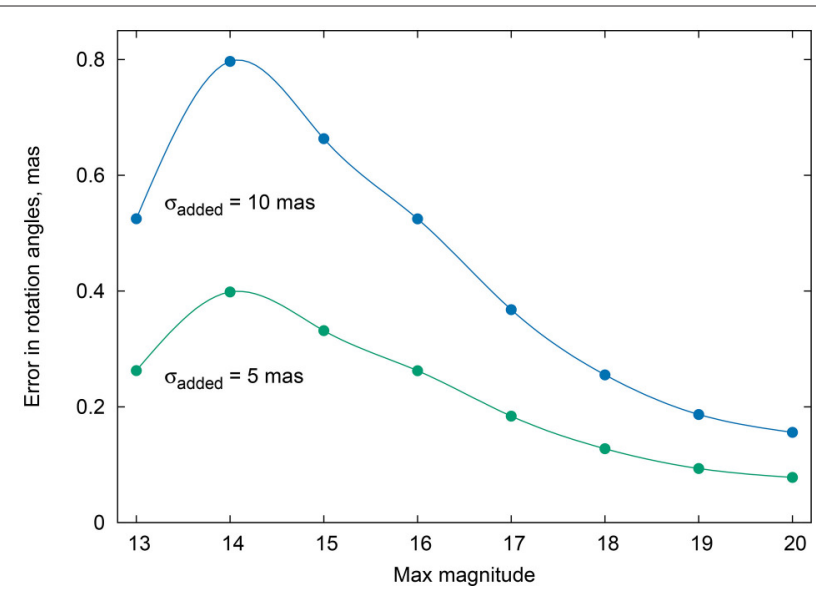

FIGURE 8 | Simulated error in orientation angles depending on the additive error due to radio-optics position shift. antenna of the VLBA (Very Long Baseline Array) narrow regional network. The position of radio stars were determined by means of phase referencing to close ICRF sources with an error of about 10 mas on average. The HCRF-ICRF orientation angles were estimated with an error of $\sim 2.7$ mas.

The current accuracy of both VLBI and optical (Gaia) observations is much better. Consequently, properly scheduled radio star observations using large regional or global VLBI networks can provide much smaller position errors and hence better accuracy of the link between optical and radio frames. A Monte Carlo simulation was performed by Malkin (2016a) to estimate an error in the orientation angle between GCRF and ICRF obtained from radio stars observation. Results of this study showed that VLBI observations of radio stars can provide an independent and accurate method to link the GCRF to the ICRF. A properly organized VLBI program for radio star observations can lead to the realization of the GCRF-ICRF link with an error of about 0.1 mas with a reasonable load on the VLBI network. Thus, this method can provide a valuable contribution to the improvement of the GCRF-ICRF link.

Details of this work are given in Malkin (2016a).

\section{GALACTIC ABERRATION}

Comparison of the ICRF and GCRF catalogs should be made at a certain epoch $t_{0}$. Two natural choices are the current standard epoch of the astronomical equations and quantities $t_{0}=\mathrm{J} 2000.0$, and the mean epoch of the Gaia catalog $t_{0}=\sim 2017.0$, supposing a 5-year period of Gaia operations starting from July 2014. It can be reasonably supposed that the Gaia positions will be brought to $t_{0}$ using its own proper motions.

The situation with the ICRF positions is not so simple. The ICRF concept is based on the absence of detectable motions of ICRF sources. It is definitely not the case at the micro-arcsecond level of accuracy. Several works showed that astrometric VLBI observations are capable of revealing statistically meaningful apparent motions of the ICRF objects, see, e.g., MacMillan (2005)

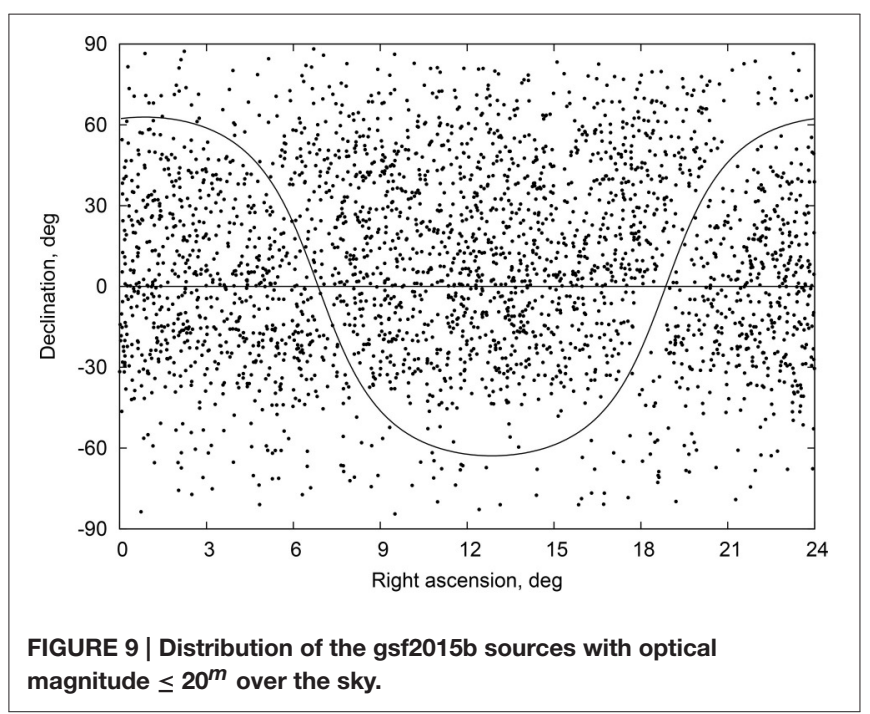


and Titov and Lambert (2013). The question of the nature of these motions is very complicated, and the consistency between various estimates is not satisfactory yet. The overall problem of source motions is beyond the scope of this paper. Here, only one systematic component of source motion pattern, namely galactic aberration in proper motions (GA) is discussed. The theory of this effect is considered in Kovalevsky (2003), Kopeikin and Makarov (2006), Liu et al. (2012), and Liu et al. (2013). The proper motion caused by the GA is given by Malkin (2011):

$$
\begin{aligned}
\mu_{l} \cos b & =-A \sin l, \\
\mu_{b} & =-A \cos l \sin b,
\end{aligned}
$$

where $l$ and $b$ are the galactic longitude and latitude of the object, respectively, and $A$ is the GA constant depending on the galactic rotation parameters. The most probable value of the GA constant is $A=5.0 \pm 0.3 \mu \mathrm{as} / \mathrm{yr}$ (Malkin, 2014).

To estimate the impact of the GA on the orientation angles between ICRF and GCRF, a special test was performed. The idea of this test was proposed by Malkin (2015b)and is extended here. Two catalogs of radio source positions were used for the simulation. The first catalog comprises 688 ICRF2 sources of AGN type and with visual magnitude $18^{m}$ or brighter following the principles of the link source selection proposed by Bourda et al. (2008). The second catalog consists of 2815 gsf2015b sources discussed in Section 4.

All the existing catalogs of radio source positions are derived without accounting for the GA during data processing. Therefore, to bring the selected link source positions to the epoch $t_{0}$ the following equations should be used:

$$
\begin{aligned}
\alpha\left(t_{0}\right) & =\alpha(t)-\mu_{\alpha}\left(t-t_{0}\right), \\
\delta\left(t_{0}\right) & =\delta(t)-\mu_{\delta}\left(t-t_{0}\right),
\end{aligned}
$$

where $t$ is the mean epoch of observations of the source in the ICRF2 or GSFC catalogs, $\alpha(t)$ and $\delta(t)$ are source coordinates (right ascension and declination, respectively) in the catalog, $\mu_{\alpha}$ and $\mu_{\delta}$ are GA-induced motions in right ascension and declination, respectively, computed by Equation (5) and transformed to the equatorial system as described by Malkin (2014) and Malkin (2015a). All the computations were made for $t_{0}=\mathrm{J} 2000.0$ and $t_{0}=2017.0$.

Then, we have two catalogs for each of four variants (two initial catalogs and two $t_{0}$ epochs). The first catalog in each pair is merely the initial catalog. Such a catalog would be used for the ICRF-GCRF link if GA is not taken into account, which is currently the case. The second catalog contains positions of the same sources transferred to the epoch $t_{0}$ for the GA-induced proper motions. This catalog would correspond to the radio source positions computed with taking into account the GA effect during VLBI data processing. Consequently the orientation angles between the two catalogs were computed. Results are presented in Table 2.

The first line of Table 2 corresponds to computations made in Malkin (2015b). The three other lines are the test extension that allowed us to correct the earlier preliminary conclusion. After the preliminary test (first line) a conclusion was drawn
TABLE 2 | Impact of galactic aberration on the orientation angles between the ICRF and GCRF.

\begin{tabular}{lcrrc}
\hline \multicolumn{1}{c}{ Catalog } & $\boldsymbol{t}_{\mathbf{0}}$ & \multicolumn{1}{c}{$\boldsymbol{A}_{\mathbf{1}}$} & \multicolumn{1}{c}{$\boldsymbol{A}_{\mathbf{2}}$} & \multicolumn{1}{c}{$\boldsymbol{A}_{\mathbf{3}}$} \\
\hline ICRF2 & $\mathrm{J} 2000.0$ & $1.3 \pm 0.5$ & $-0.1 \pm 0.5$ & $0.3 \pm 0.4$ \\
& 2017.0 & $26.8 \pm 2.4$ & $-4.9 \pm 2.3$ & $4.0 \pm 2.0$ \\
gsf2015b & $\mathrm{J} 2000.0$ & $-19.4 \pm 0.5$ & $1.9 \pm 0.5$ & $0.6 \pm 0.4$ \\
& 2017.0 & $36.1 \pm 0.6$ & $-4.2 \pm 0.6$ & $2.7 \pm 0.6$ \\
\hline
\end{tabular}

Unit: $\mu$ as.

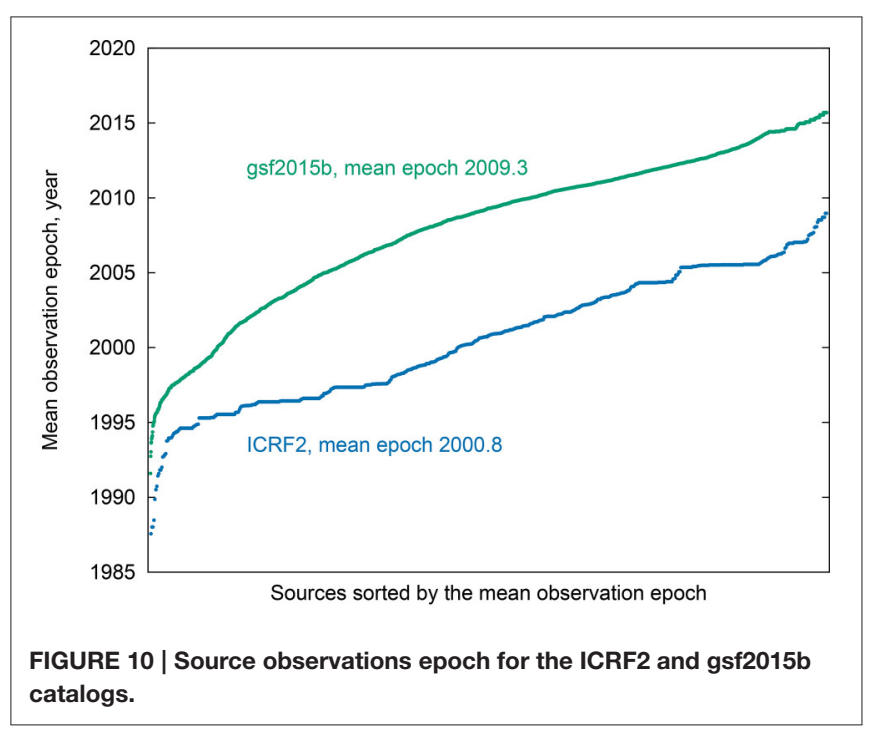

that the impact of the GA on the ICRF-GCRF link is practically negligible. More detailed test performed in this study has shown that the GA effect can be substantial. This can be seen from both the significant value of the orientation angles and their uncertainties. The errors in the orientation angles depend on both the number of common sources in the catalogs and the time interval between the mean source observation epoch in the catalog (let us call it catalog epoch) and $t_{0}$. Mean epochs for two catalogs are shown in Figure 10. The ICRF2 catalog epoch is closer to J2000.0 than the gsf2015b epoch, but the latter contains four times more sources, which resulted in about the same errors in the orientation angles for $t 0=\mathrm{J} 2000$.0. In contrast, the gsf2015b catalog epoch is closer to 2017.0, which together with larger number of sources gives much smaller errors in the orientation angles as compared with the ICRF2.

\section{DISCUSSION AND CONCLUSION}

A great milestone in the construction of the celestial reference frame is the Gaia mission, which will result in the GCRF with expected accuracy of a few tens of micro-arcsecond for final release in the early 2020s. A new ICRF release based on VLBI observations of extragalactic radio sources of similar accuracy can be also expected by that time. Constructing of a single multifrequency celestial reference frame based on the ICRF and GCRF 
is the primary task of fundamental astronomy for the next decade (Gaume, 2015).

The first step toward this goal is an accurate alignment of the Gaia catalog to the ICRF. The desired goal is to achieve mutual orientation between the two frames with an accuracy of 0.1 mas or better, which is a challenging task.

Currently, the International VLBI Service for Geodesy and Astrometry (IVS) is conducting a special program on observation of prospective link sources not having a sufficient number of observations in the framework of regular observing programs (Le Bail et al., 2016). This forms part of the plan to prepare to achieve the GCRF-ICRF link using 195 selected sources selected on basis of several criteria, such as, inclusion in the ICRF2, optical magnitude $\leq 18^{m}$, symmetric compact structure, sufficient radio flux density. The two first criteria seem to be outdated. Firstly, the ICRF2 is currently not the most appropriate radio source position catalog that can serve as an ICRF3 prototype, which is planned to be used for initial alignment of the Gaia catalog to the ICRS as discussed in Section 4. Secondly, as shown in Section 5, using optically fainter sources up to $20^{\mathrm{m}}$ provides more precise determination of the orientation angles. It is even more important that the use of more sources with reliable VLBI positions is necessary to mitigate the impact of most of the negative factors mentioned below, see Figure 8 and related text. As discussed, there are possibilities to multiply the number of link sources taking into account the substantial increase of the number of radio sources with reliably determined positions and the number of sources having photometry measurements.

The following problems were identified in the literature that dilute the accuracy of the link between radio and optical frames:

- Structure effects (discussed below).

- Systematic errors of radio position catalogs.

- Multiple radio sources related to a single object in optics, e.g., binary black holes, and vice versa.

- Gravitational lenses.

- Errors in ICRF-GCRF cross-identification.

Most probably, the main factor that will deteriorate the accuracy of the link between the radio and optical frames, is the source structure. It can manifest at both radio and optical wavelengths as complex, asymmetric distribution of the brightness over the source map, spatial bias between the optical and radio brightness centroids, core-shift effects, spatial bias between the core/AGN brightness centroid and with respect to the optical centroid of the host galaxy. Moreover, the structure effects are often variable. Although many studies are devoted to this problem, see, Fey and Charlot (1997); da Silva Neto et al. (2002); Moór et al. (2011); Bouffet et al. (2013); Zacharias and Zacharias (2014); Berghea et al. (2016) and papers cited therein, there are insufficient data to quantify the impact of source structure on the accuracy of the orientation angles between optical and radio frames. Evidently, the most complete study is provided by Zacharias and Zacharias (2014) used in the current work. It should be noted that though the structure effect can reach several mas for an individual source, it can hardly be systematic and thus will be averaged over the sky during the computation of the orientation angles between the
GCRF and ICRF. However, supplementary observations and theoretical considerations are needed to quantify this effect more accurately.

Two main methods to obtain the link between the GCRF and ICRF were considered in this paper. The first method is direct Gaia observations of the sufficiently optically bright ICRF sources. This method allows for a straightforward solution of the task. However, there are serious constraints on the accuracy of this method caused by the previously mentioned factors. These factors can limit the real accuracy of the GCRF-ICRF link to 0.1 mas or worse.

Observations of radio stars can serve as an alternative equally accurate method. It was successfully used to link the HCRF to the ICRF. However, this method is also affected by some accuracylimiting factors (Malkin, 2016a). Many radio stars comprise double or multiple systems, and thus their orbital motions must be accounted for. The accurate Gaia-derived orbits can be used for this purpose. Moreover, radio stars may have complex and variable structures, which might cause a time-dependent bias between the radio and optical positions. Lestrade et al. (1995) estimated the impact of radio star structure and possible variations in the radio star emitting center to be within the error budget of $\sim 0.5$ mas. Lestrade et al. (1999) found that the structure-induced systematic errors in the VLBI positions of 12 stars ranged from 0.07 mas to 0.54 mas, with a median value of 0.18 mas. Provided that several tens of radio stars have been observed, this factor should not significantly impact on the errors in the orientation angles.

Combination of two methods of linking the GCRF to the ICRF should facilitate improvement of the systematic accuracy of the link between radio and optical frames.

Finally, it should be noted that the preparation for the aligning of the Gaia catalog to ICRF3 planned for 2018-2019 is only an intermediate stage in construction of the multi-frequency celestial reference frame. Certainly, the main work on the link between radio and optical frames is to be done in the early 2020s, after the final Gaia catalog is prepared. It is desirable to plan preparation of a new ICRF realization, will it be called ICRF4 or ICRF3 extension, at the same time, i.e., immediately before the final GCRF link to ICRF. Comparison of gsf2015b with ICRF2 has clearly shown that the latest radio catalog should be used for this work due to the accuracy of the VLBI-derived CRF solutions which rapidly improves with time. In the framework of this activity, it appears to be very important to fast-track the plans on the ICRF improvements in the Southern Hemisphere (Jacobs et al., 2014; Plank et al., 2015), and to start a program of observations of radio stars (Malkin, 2016a).

As the Hipparcos experience has shown, it can be expected that the work on improving the link between radio and optical frames will be continued during a long period after completing the Gaia mission. Continuous quality improvement of a VLBI-based ICRF promises corresponding improvement of this link with time.

\section{AUTHOR CONTRIBUTIONS}

The author confirms being the sole contributor of this work and approved it for publication. 


\section{ACKNOWLEDGMENTS}

The author would like to thank the two reviewers and the handling editor for their valuable help in improving the

\section{REFERENCES}

Andrei, A. H., Anton, S., Barache, C., Bouquillon, S., Bourda, G., Le Campion, J.F., et al. (2012). Gaia initial QSO catalogue: the variability and compactness indexes. Mem. Soc. Astron. 83:930.

Andrei, A. H., Antón, S., Taris, F., Bourda, G., Souchay, J., Bouquillon, J., et al. (2014). “The Gaia initial quasar catalog," in Journées 2013 "Systèmes de référence spatio-temporels”, ed N. Capitaine (Paris: Paris Observatory), 84-87.

Andrei, A. H., Taris, F., Anton, S., Bourda, G., Damljanovic, G., Souchay, J., et al. (2015). A complete bank of optical images of the ICRF QSOs. IAU Gen. Assem. 22:2243437.

Arias, E. F., Charlot, P., Feissel, M., and Lestrade, J.-F. (1995). The extragalactic reference system of the International Earth Rotation Service, ICRS. Astron. Astrophys. 303, 604-608.

Berghea, C. T., Makarov, V. V., Frouard, J., Hennessy, G. S., Dorland, B. N., Veillette, D. R., et al. (2016). A Global Astrometric Solution for Pan-STARRS referenced to ICRF2. arXiv:1606.03446.

Boboltz, D. A., Fey, A. L., Puatua, W. K., Zacharias, N., Claussen, M. J., Johnston, K. J., et al. (2007). Very large array plus pie town astrometry of 46 radio stars. Astron. J. 133, 906-916. doi: 10.1086/510154

Bouffet, R., Charlot, P., and Lambert, S. (2013). "Radio source structure and VLBI position instabilities," in SF2A-2013: Proceedings of the Annual Meeting of the French Society of Astronomy and Astrophysics, eds L. Cambresy, F. Martins, E. Nuss, and A. Palacios (Montpelier, VT), 161-164.

Bourda, G., Charlot, P., and Le Campion, J.-F. (2008). Astrometric suitability of optically-bright ICRF sources for the alignment with the future Gaia celestial reference frame. Astron. Astrophys. 490, 403-408. doi: 10.1051/00046361:200810667

Bourda, G., Charlot, P., Porcas, R. W., and Garrington, S. T. (2010). VLBI observations of optically-bright extragalactic radio sources for the alignment of the radio frame with the future Gaia frame. I. Source detection. Astron. Astrophys. 520:A113. doi: 10.1051/0004-6361/201014248

Bourda, G., Collioud, A., Charlot, P., Porcas, R., and Garrington, S. (2011). VLBI observations of optically-bright extragalactic radio sources for the alignment of the radio frame with the future Gaia frame. II. Imaging candidate sources. Astron. Astrophys. 526:A102. doi: 10.1051/0004-6361/201014249

da Silva Neto, D. N., Andrei, A. H., Vieira Martins, R., and Assafin, M. (2002). A pattern of noncoincidence between radio and optical positions of international celestial reference frame sources. Astron. J. 124, 612-618. doi: 10.1086/341163

de Bruijne, J. H. J., Rygl, K. L. J., and Antoja, T. (2014). Gaia astrometric science performance - post-launch predictions. EAS Publ. Ser. 67, 23-29. doi: $10.1051 /$ eas/1567004

de Souza, R. E., Krone-Martins, A., dos Anjos, S., Ducourant, C., and Teixeira, R. (2014). Detection of galaxies with Gaia. Astron. Astrophys. 568:A124. doi: 10.1051/0004-6361/201423514

Fey, A. L., and Charlot, P. (1997). VLBA observations of radio reference frame sources. II. Astrometric suitability based on observed structure. Astrophys. J. Suppl. Ser. 111, 95-142.

Fey, A. L., Gordon, D., Jacobs, C. S., Ma, C., Gaume, R. A., Arias, E. F., et al. (2015). The Second Realization of the International Celestial Reference Frame by Very Long Baseline Interferometry. Astron. J. 150:58. doi: 10.1088/0004$6256 / 150 / 2 / 58$

Gaume, R. (2015). The IAU Division A Working Group on the Third Realization of the ICRF: Background, Goals, Plans. IAU Gen. Assem. 22:2256777.

Gordon, D., Jacobs, C., Beasley, A., Peck, A., Gaume, R., Charlot, P., et al. (2016). Second epoch VLBA calibrator survey observations: VCS-II. Astron. J. 151:154. doi: 10.3847/0004-6256/151/6/154

Jacobs, C. S., Arias, F., Boboltz, D., Boehm, J., Bolotin, S., Bourda, G., et al. (2014). "ICRF-3: roadmap to the next generation ICRF," in Proceeding Journées 2013 Systèmes de Référence Spatio-temporels, Observatoire de Paris, ed N. Capitaine (Paris), 51-56. manuscript. This work was partially supported by the Russian Government Program of Competitive Growth of Kazan Federal University. This research has made use of NASA's Astrophysics Data System.

Kopeikin, S., and Makarov, V. (2006). Astrometric effects of secular aberration. Astron. J. 131, 1471-1478. doi: 10.1086/500170

Kovalevsky, J. (2003). Aberration in proper motions. Astron. Astrophys. 404, 743-747. doi: 10.1051/0004-6361:20030560

Kovalevsky, J., Lindegren, L., Perryman, M. A. C., Hemenway, P. D., Johnston, K. J., Kislyuk, V. S., et al. (1997). The HIPPARCOS catalogue as a realisation of the extragalactic reference system. Astron. Astrophys. 323, 620-633.

Le Bail, K., Gipson, J. M., Gordon, D., MacMillan, D. S., Behrend, D., Thomas, C. C., et al. (2016). IVS observation of ICRF2-Gaia transfer sources. Astron. J 151:79. doi: 10.3847/0004-6256/151/3/79

Lestrade, J.-F., Jones, D. L., Preston, R. A., Phillips, R. B., Titus, M. A., Kovalevsky, J., et al. (1995). Preliminary link of the HIPPARCOS and VLBI reference frames. Astron. Astrophys. 304:182.

Lestrade, J.-F., Preston, R. A., Jones, D. L., Phillips, R. B., Rogers, A. E. E., Titus, M. A., et al. (1999). High-precision VLBI astrometry of radio-emitting stars. Astron. Astrophys. 344, 1014-1026.

Lindegren, L., Babusiaux, C., Bailer-Jones, C., Bastian, U., Brown, A. G. A., Cropper, M., et al. (2008). "The Gaia mission: science, organization and present status," in IAU Symposium, Vol. 248, eds W. J. Jin, I. Platais, and M. A. C. Perryman (Shanghai), 217-223.

Lindegren, L., and Kovalevsky, J. (1995). Linking the HIPPARCOS Catalogue to the extragalactic reference system. Astron. Astrophys. 304:189.

Liu, J.-C., Capitaine, N., Lambert, S. B., Malkin, Z., and Zhu, Z. (2012). Systematic effect of the Galactic aberration on the ICRS realization and the Earth orientation parameters. Astron. Astrophys. 548:A50. doi: 10.1051/0004$6361 / 201219421$

Liu, J.-C., Xie, Y., and Zhu, Z. (2013). Aberration in proper motions for stars in our Galaxy. Mon. Not. Roy. Astron. Soc. 433, 3597-3604. doi: $10.1093 / \mathrm{mnras} / \mathrm{stt} 1006$

MacMillan, D. S. (2005). "Quasar apparent proper motion observed by geodetic VLBI networks," in Future Directions in High Resolution Astronomy, volume 340 of Astronomical Society of the Pacific Conference Series, eds J. Romney and M. Reid (San Francisco, CA: Astronomical Society of the Pacific), 477-481.

Malkin, Z. (2014). On the implications of the Galactic aberration in proper motions for the Celestial Reference Frame. Mon. Not. Roy. Astron. Soc. 445, 845-849. doi: $10.1093 / \mathrm{mnras} / \mathrm{stu} 1796$

Malkin, Z. (2015a). Erratum: on the implications of the Galactic aberration in proper motions for celestial reference frame. Mon. Not. R. Astron. Soc. 447, 4028. doi: 10.1093/mnras/stv032

Malkin, Z. (2015b). How much can Galactic aberration impact the link between radio (ICRF) and optical (GCRF) reference frames. arXiv:1509.07245.

Malkin, Z. (2016a). Using radio stars to link the Gaia and VLBI reference frames. Mon. Not. R. Astron. Soc. 461, 1937-1942. doi: 10.1093/mnras/stw1488

Malkin, Z. (2016b). Second version of the catalog of optical characteristics of astrometric radio sources OCARS. Astron. Rep. 60. doi: 10.1134/S106377 2916110032

Malkin, Z. M. (2011). The influence of Galactic aberration on precession parameters determined from VLBI observations. Astron. Rep. 55, 810-815. doi: $10.1134 /$ S1063772911090058

Mignard, F. (2014). "Building a CRF with gaia," in Dynamical Astronomy in Latin-America - 2014. Available online at: http://adela2014.das.uchile.cl/talks/ Workshop_Classes/FMignard_Lecture_2_ADeLA2014@SCL.pdf

Moór, A., Frey, S., Lambert, S. B., Titov, O. A., and Bakos, J. (2011). On the connection of the apparent proper motion and the VLBI structure of compact radio sources. Astron. J. 141, 178. doi: 10.1088/0004-6256/141/6/178

Perryman, M. A. C., de Boer, K. S., Gilmore, G., Høg, E., Lattanzi, M. G., Lindegren, L., et al. (2001). GAIA: composition, formation and evolution of the Galaxy. Astron. Astrophys. 369, 339-363. doi: 10.1051/0004-6361:20010085

Plank, L., Lovell, J. E. J., Shabala, S. S., Böhm, J., and Titov, O. (2015). Challenges for geodetic VLBI in the southern hemisphere. Adv. Space Res. 56, 304-313. doi 10.1016/j.asr.2015.04.022 
Sokolova, J., and Malkin, Z. (2007). On comparison and combination of catalogues of radio source positions. Astron. Astrophys. 474, 665-670. doi: 10.1051/00046361:20077450

Souchay, J., Andrei, A. H., Barache, C., Kalewicz, T., Gattano, C., Coelho, B., et al. (2015). The third release of the Large Quasar Astrometric Catalog (LQAC-3): a compilation of 321957 objects. Astron. Astrophys. 583:A75. doi: 10.1051/0004-6361/201526092

Taris, F., Andrei, A., Klotz, A., Vachier, F., Côte, R., Bouquillon, S., et al. (2013). Optical monitoring of extragalactic sources for linking the ICRF and the future Gaia celestial reference frame. I. Variability of ICRF sources. Astron. Astrophys. 552:A98. doi: 10.1051/0004- 6361/201219686

Taris, F., Andrei, A., Roland, J., Klotz, A., Vachier, F., and Souchay, J. (2016). Longterm $\mathrm{R}$ and $\mathrm{V}$-band monitoring of some suitable targets for the link between ICRF and the future Gaia celestial reference frame. Astron. Astrophys. 587:A112. doi: 10.1051/0004-6361/201526676

Taris, F., Damljanovic, G., Andrei, A., Klotz, A., and Vachier, F. (2015). "Optical monitoring of QSO in the framework of the Gaia space mission," in Journées 2014 "Systèmes de référence spatio-temporels", eds Z. Malkin and N. Capitaine, 42-43.

Titov, O., and Lambert, S. (2013). Improved VLBI measurement of the solar system acceleration. Astron. Astrophys. 559:A95. doi: 10.1051/0004-6361/201321806
Véron-Cetty, M.-P., and Véron, P. (2006). A catalogue of quasars and active nuclei: 12th edition. Astron. Astrophys. 455, 773-777. doi: 10.1051/00046361:20065177

Zacharias, N., Finch, C., Subasavage, J., Bredthauer, G., Crockett, C., Divittorio, M., et al. (2015). The first U.S. naval observatory robotic astrometric telescope catalog. Astron. J. 150:101. doi: 10.1088/0004-6256/15 $0 / 4 / 101$

Zacharias, N., and Zacharias, M. I. (2014). Radio-optical reference frame link using the U.S. naval observatory astrograph and deep CCD imaging. Astron. J. 147:95. doi: 10.1088/0004-6256/147/5/95

Conflict of Interest Statement: The author declares that the research was conducted in the absence of any commercial or financial relationships that could be construed as a potential conflict of interest.

Copyright (C) 2016 Malkin. This is an open-access article distributed under the terms of the Creative Commons Attribution License (CC BY). The use, distribution or reproduction in other forums is permitted, provided the original author (s) or licensor are credited and that the original publication in this journal is cited, in accordance with accepted academic practice. No use, distribution or reproduction is permitted which does not comply with these terms. 